\title{
Approximated Context-Sensitive Analysis for Parameterized Verification
}

\author{
Parosh Aziz Abdulla ${ }^{1}$, Giorgio Delzanno ${ }^{2}$, and Ahmed Rezine ${ }^{3}$ \\ ${ }^{1}$ Uppsala University, Sweden \\ parosh@it.uu.se \\ ${ }^{2}$ Università di Genova, Italy \\ giorgio@disi.unige.it \\ ${ }^{3}$ University of Paris 7, France \\ rezine.ahmed@liafa.jussieu.fr
}

\begin{abstract}
We propose a verification method for parameterized systems with global conditions. The method is based on context-sensitive constraints, a symbolic representation of infinite sets of configurations defined on top of words over a finite alphabet. We first define contextsensitive constraints for an exact symbolic backward analysis of parameterized systems with global conditions. Since the model is Turing complete, such an analysis is not guaranteed to terminate. To turn the method into a verification algorithm, we introduce context-sensitive constraints that over-approximate the set of backward reachable states and show how to symbolically test entailment and compute predecessors. We apply the resulting algorithm to automatically verify parameterized models for which the exact analysis and other existing verification methods either diverge or return false positives.
\end{abstract}

\section{Introduction}

We consider verification of safety properties for parameterized systems with universal and existential global conditions. Typically, such a system consists of an arbitrary number of processes organized in a linear array. Global conditions are used as guards. An example of a universally quantified global condition is that all processes to the left of a given process $i$ should satisfy a property $\varphi$. Process $i$ can perform the transition only if all processes with indices $j<i$ satisfy $\varphi$. In an existential condition we require that some (rather than all) processes satisfy $\varphi$. The task is to verify correctness regardless of the number of processes.

In 3 we have proposed a light-weight verification method for parameterized systems based on monotonic abstraction with the aim of avoiding the use of the full power of automata and regular languages (which require heavy manipulations like the use of transducers [21/14/7/9]). The main idea of the method in [3] is to consider a transition relation that is an over-approximation of the one induced by the parameterized system. To do that, we modify the semantics of universal quantifiers by eliminating the processes that violate the given condition (downward closed semantics). The obtained approximate transition 
system is monotonic with respect to the subword relation (larger configurations are able to simulate smaller ones). Since the approximate transition relation is monotonic, it can be analyzed using a symbolic backward reachability algorithm based on a generic method introduced in [2]. The algorithm operates on upward closed sets of configurations (with respect to the subword relation) and uses symbolic operations that are much simpler than transducers and regular languages. The PFS tool [3] that implements this technique can thus be applied to verify safety properties for configurations with any number of processes. Monotonic abstraction has proven successful in verifying a wide range of parameterized, distributed, and heap manipulating systems 35461]. However, it may return false positives due to a loss of precision in the representation of special witness processes. We give an example of a system where such a situation occurs.

An example in which monotonic abstraction may return false positives is the parameterized system where processes are represented in Fig. 1, Each process has five local states $q_{0}, \ldots, q_{4}$. All processes are initially in state $q_{0}$. A process in the critical section is at state $q_{4}$. Note that the set of configurations violating mutual exclusion contains exactly configurations with at least two occurrences of symbol $q_{4}$. Processes start crossing from $q_{0}$ to $q_{1}$, and then to state $q_{2}$.

Once the first process has crossed to state $q_{2}$ it "closes the door" on the processes which are still in $q_{0}$. These processes will no longer be able to leave $q_{0}$ until the door is opened again (when no process is in state $q_{2}$ or $\left.q_{3}\right)$. Furthermore, a process is allowed to cross from $q_{3}$ to state $q_{4}$ only if there is at least one process still in state $q_{2}$ (i.e., the door is still closed on the processes in state $q_{0}$ ).

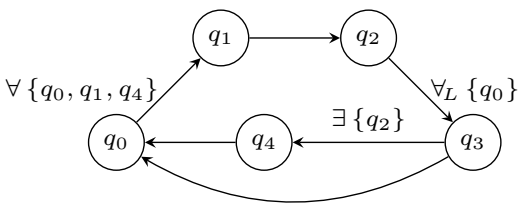

Fig. 1. State diagram of an individual process This is to prevent a process first reaching

$q_{4}$ and then a process to its left starting to move from $q_{0}$ all the way to state $q_{4}$ (thus violating mutual exclusion). From the set of processes which have left state $q_{0}$ (and which are now in state $q_{1}$ or $q_{2}$ ) the leftmost process has the highest priority. This is encoded by the global condition that a process may move from $q_{2}$ to $q_{3}$ only subject to the global condition that all processes to its left are in state $q_{0}$ (this condition is encoded by the universal quantifier $\forall_{L}$, where "L" stands for "Left"). A typical run of the system is of the form $q_{0} q_{0} q_{0} q_{0} \longrightarrow q_{0} q_{1} q_{0} q_{0} \longrightarrow q_{0} q_{1} q_{1} q_{0} \longrightarrow q_{0} q_{2} q_{1} q_{0} \longrightarrow q_{0} q_{2} q_{2} q_{0} \longrightarrow q_{0} q_{3} q_{2} q_{0} \longrightarrow$ $q_{0} q_{4} q_{2} q_{0} \longrightarrow q_{0} q_{0} q_{2} q_{0}$. The protocol satisfies mutual exclusion. Consider now the abstract transition system computed by applying monotonic abstraction. From the next-to-last configuration, the left most process can move (in the abstract system) to $q_{1}$. More precisely, the run may continue as follows in the abstract system. $q_{0} q_{4} q_{2} q_{0} \longrightarrow q_{1} q_{4} q_{0} \longrightarrow q_{2} q_{4} q_{0} \longrightarrow q_{3} q_{4} q_{0} \longrightarrow q_{4} q_{4} q_{0}$. Notice that monotonic abstraction removes the guard (the process in state $q_{2}$ ) since it does not satisfy the global condition of the rule $q_{0} \rightarrow q_{1}: \forall\left\{q_{0}, q_{1}, q_{4}\right\}$. With this 
abstraction the door is opened again. This allows processes in $q_{0}$ to move again, enabling one of them to reach $q_{4}$. This gives a false positive.

This kind of false positives arise typically in systems where correctness depends on the existence of a witness process. For this reason, it is relevant to study new approximations that can be used for more precise analysis than that provided by monotonic abstraction. The challenge here is to preserve the positive features of the latter approach such as the use of simple data structures and of a generic verification algorithm based on well-quasi orderings.

New Contribution. We propose a new verification algorithm based on an approximated context sensitive analysis that improves the precision of monotonic abstraction. The method is guaranteed to terminate, and is based on relatively simple symbolic data structures. We build the verification method in two steps.

We first define a symbolic representation, namely context-sensitive constraints, that are a natural generalization of the constraints used in the monotonic abstraction framework. In monotonic abstraction a word $w$ of process states (referred to as the basis) is used as a symbolic representation of its upward closure computed with respect to word inclusion. This implies that any type of processes is allowed in between two consecutive states of the basis $w$ (these allowed processes are referred to as context). Context-sensitive constraints generalize this idea by introducing constraints on the type of processes that are allowed to occur in each context. For each pair of consecutive states in the basis, constraints are expressed by using a subset $R$ of states: only processes with states in $R$ are allowed in this context. This kind of constraints can be used to exactly represent (one-step) predecessor configurations of a parameterized system with global conditions. An analysis based on this kind of constraints is not guaranteed to terminate in general. Furthermore, when testing in practice, even on simple examples the number of generated constraints often explodes after a few steps. Therefore, approximations are necessary to ensure both theoretical (e.g. using wqo theory) and practical termination (e.g. using more compact representations).

The approximated method we propose in this paper works on constraints of a special form, called simple context-sensitive constraints. In a simple contextsensitive constraint we use a single subset of states, called the padding set, to over-approximate the constraints on processes in each context. For this new symbolic representation, we have the following properties. The entailment ordering turns out to be a well-quasi ordering. The computation of predecessors is guaranteed to terminate and to return a finite representation of an over-approximation of the exact set of predecessor configurations. Our abstract predecessor operator incorporates accelerations in the computation of predecessors for ordered system that are similar in spirit to widening operators used in the unordered case (as those used in relation analysis for counter systems e.g. in [12 27/28]). Finally, the constraint operations are much simpler and more efficient than those used in the exact context-sensitive analysis. Since simple context-sensitive constraints can represent upward closed sets of configuration computed with respect to word inclusion, the resulting over-approximation is guaranteed to be at least as precise 
as monotonic abstraction. However, in several practical examples it gives more precise results (eliminates false positives).

As a first set of experiments, we have considered benchmark examples of parameterized systems taken from [15/7/3 . The performance of the new verification algorithm is comparable with that of the PFS tool based on monotonic abstraction 3. We remark that in these examples exact analysis often diverges or suffers from the symbolic state explosion problem. Furthermore, we also consider several new case-studies that include both ordered systems like formulations of Szymanski's algorithm with non-atomic updates (semi-automatically verified in [182223]), and unordered concurrent systems like synchronization skeletons [12 27/28] and reference counting schemes for virtual memory [16]. For these examples monotonic abstraction often returns spurious error traces due to a loss of precision in the representation of special processes (as in Szymanski) or in the representation of counters. Our new verification algorithm eliminates all the false positives and verifies the new case studies for any number of processes/unbounded value of counters. We are not aware of other tools that can automatically verify the same class of ordered/unordered parameterized models.

Plan of the paper. We describe our model of parameterized systems in the next Section. Then, we introduce context-sensitive constraints in Section 3, and simple-context sensitive constraints in Section 4. In Section 5, we discuss experimental results. Finally, in Section 6 we discuss related and future work.

\section{Model}

For a set $A$, we use $A^{*}$ to denote the set of finite words over $A$, and use $w_{1} w_{2}$ to denote the concatenation of two words $w_{1}$ and $w_{2}$ in $A^{*}$. For a natural number $n$, we use $\bar{n}$ to denote the set $\{1, \ldots, n\}$.

Formally, a parameterized system is a pair $\mathcal{P}=(Q, T)$, where $Q$ is a finite set of local states, and $T$ is a finite set of transitions. A transition is either local or global. A local transition is of the form $q \rightarrow q^{\prime}$, where a process changes state from $q$ to $q^{\prime}$ independently of the states of the other processes. A global transition is of the form $q \rightarrow q^{\prime}: \mathbb{Q} P$, where $\mathbb{Q} \in\left\{\exists_{L}, \exists_{R}, \exists_{L R}, \forall_{L}, \forall_{R}, \forall_{L R}\right\}$ and $P \subseteq Q$. Here, the process checks the states of the other processes. For instance, the condition $\forall_{L} P$ means "all processes to the left are in states belonging to $P$ "; the condition $\forall_{L R} P$ means "all other processes (whether to the left or to the right) are in states belonging to $P$ "; and so on.

A parameterized system $\mathcal{P}=(Q, T)$ induces an infinite-state transition system $(C, \longrightarrow)$ where $C=Q^{*}$ is the set of configurations and $\longrightarrow$ is a transition relation on $C$. For a configuration $c=q_{1} q_{2} \cdots q_{n}$, we define $c^{\bullet}:=\left\{q_{1}, \ldots, q_{n}\right\}$. For configurations $c=c_{1} q c_{2}, c^{\prime}=c_{1} q^{\prime} c_{2}$, and a transition $t \in T$, we write $c \stackrel{t}{\longrightarrow} c^{\prime}$ to denote that one of the following conditions is satisfied:

$-t$ is a local transition of the form $q \rightarrow q^{\prime}$.

$-t$ is a global transition of the form $q \rightarrow q^{\prime}: \mathbb{Q} P$, and one of the following conditions is satisfied: 
- either $\mathbb{Q} P=\exists_{L} P$ and $c_{1} \bullet P \neq \emptyset, \mathbb{Q} P=\exists_{R} P$ and $c_{2} \bullet \cap \neq \emptyset$, or $\mathbb{Q} P=\exists_{L R} P$ and $\left(c_{2} \bullet \cup c_{2} \bullet\right) \cap P \neq \emptyset$.

- either $\mathbb{Q} P=\forall_{L} P$ and $c_{1} \bullet \subseteq P, \mathbb{Q} P=\forall_{R} P$ and $c_{2} \bullet \subseteq P$, or $\mathbb{Q} P=\forall_{L R} P$ and $\left(c_{1}^{\bullet} \cup c_{2} \bullet\right) \subseteq P$.

We use $\stackrel{*}{\longrightarrow}$ to denote the reflexive transitive closure of $\longrightarrow$.

We define an ordering $\preceq$ on configurations as follows. Let $c=q_{1} \cdots q_{m}$ and $c^{\prime}=q_{1}^{\prime} \cdots q_{n}^{\prime}$ be configurations. Then, $c \preceq c^{\prime}$ if $c$ is a subword of $c^{\prime}$, i.e., there is a strictly increasing injection $h$ from $\bar{m}$ to $\bar{n}$ such that $q_{i}=q_{h(i)}$ for all $i: 1 \leq i \leq n$.

Given a parameterized system, we assume that, prior to starting the execution of the system, each process is in an (identical) initial state $q_{\text {init }}$. We use Init to denote the set of initial configurations, i.e., configurations of the form $q_{\text {init }} \cdots q_{\text {init }}$ (all processes are in their initial states). The set Init is infinite.

A set of configurations $U \subseteq C$ is upward closed with respect to $\preceq$ if $c \in U$ and $c \preceq c^{\prime}$ implies $c^{\prime} \in U$. For a configuration $c$, we use $\widehat{c}$ to denote the upward closure of $c$, i.e., the set $\left\{c^{\prime} \mid c \preceq c^{\prime}\right\}$. For sets of configurations $D, D^{\prime} \subseteq C$ we use $D \longrightarrow D^{\prime}$ to denote that there are $c \in D$ and $c^{\prime} \in D^{\prime}$ with $c \longrightarrow c^{\prime}$. The coverability problem for parameterized systems is defined as follows:

\section{PAR-COV}

\section{Instance}

- A parameterized system $\mathcal{P}=(Q, T)$.

- A finite set $C_{F}$ of configurations.

Question Init $\stackrel{*}{\longrightarrow} \widehat{C_{F}}$ ?

It can be shown, using standard techniques (see e.g. [26]), that checking safety properties (expressed as regular languages) can be translated into instances of the coverability problem. Typically, $\widehat{C_{F}}$ is used to characterize sets of bad configurations which we do not want to occur during the execution of the system. The system is safe iff $\widehat{C_{F}}$ is not reachable. Therefore, checking safety properties amounts to solving PAR-COV (i.e., to the reachability of upward closed sets). In Example 1 the set of bad configurations is $\widehat{q_{4} q_{4}}$.

\section{Exact Context-Sensitive Symbolic Analysis}

Assume a parameterized system $\mathcal{P}=(Q, T)$, where $Q$ is a finite set of states. In order to finitely represent infinite sets of system configurations (e.g. configurations of arbitrary size) we use the context-sensitive constraints defined in this section. For the sake of clarity, we first present a simplified version of our constraints and then discuss extensions we use in our implementation. We work with words in $\mathbb{A}^{*}$, where $\mathbb{A}=Q \cup \mathbb{P}(Q)$ and $\mathbb{P}(Q)$ denotes the set of subsets of $Q$. We use $p, q, \ldots$ to denote states in $Q$, and $P, R, \ldots$ to denote sets of states in $\mathbb{P}(Q)$. Furthermore, for $w \in \mathbb{A}^{*}$ we use $w^{\bullet}$ to denote the union of all states in $Q$ occurring in $w$ either as one of its letters or listed in one of its sets. As an example, for $R=\left\{q_{1}, q_{2}\right\}$ we have that $\left(R q_{3} R\right)^{\bullet}=\left\{q_{1}, q_{2}, q_{3}\right\}$. 
Definition 1. A context-sensitive (CC-)constraint is a word in $\mathbb{A}^{*}$ of the form $R_{0} q_{1} R_{1} \ldots q_{n} R_{n}$, where $q_{i} \in Q$ for $i: 1 \leq i \leq n$ and $R_{i} \subseteq Q$ for $i: 0 \leq i \leq n$. The configuration $q_{1} \ldots q_{n}$ is called the basis and each set $R_{i}$ is called a context. The denotation of a context-sensitive constraint $\phi$, written $\llbracket \phi \rrbracket$, is the set of configurations of the form $c_{0} q_{1} c_{1} \ldots q_{n} c_{n}$ where $c_{i} \in R_{i}^{*}$ for $i: 0 \leq i \leq n$.

As an example, assume $Q=\left\{q_{1}, q_{2}, q_{3}\right\}, R_{0}=R_{1}=\left\{q_{2}, q_{3}\right\}$ and $R_{2}=\left\{q_{1}, q_{3}\right\}$. The constraint $\phi$ defined as $R_{0} q_{1} R_{1} q_{2} R_{2}$ denotes all configurations of the form $c_{0} q_{1} c_{1} q_{2} c_{2}$ such that sub-configurations $c_{0}$ and $c_{1}$ cannot contain processes $q_{1}$ and sub-configuration $c_{2}$ cannot contain occurrences of processes $q_{2}$. Therefore, configurations $q_{3} q_{1} q_{3} q_{2} q_{1}$ and $q_{3} q_{1} q_{3} q_{3} q_{2} q_{1}$ belong to $\llbracket \phi \rrbracket$, whereas $q_{1} q_{1} q_{2}$ and $q_{1} q_{3} q_{2} q_{2}$ do not belong to $\llbracket \phi \rrbracket$. Notice that CC's of the form $Q q_{1} Q \ldots q_{n} Q$ denote upward closed sets of states with respect to word inclusion (there are no constraints on the contexts). For instance, the set of bad states in Example 1 can be characterized by the CC $Q q_{4} Q q_{4} Q$ where $Q=\left\{q_{0}, q_{1}, q_{2}, q_{3}, q_{4}\right\}$.

We now define the symbolic operations we use in our analysis, namely the entailment and the predecessors computation on context-sensitive constraints. These respectively correspond to the application, without any loss of precision, of the inclusion and the predecessor operations on the associated denotations.

Entailment. For constraints $\phi=R_{0} q_{1} \ldots q_{n} R_{n}$ and $\phi^{\prime}=R_{0}^{\prime} q_{1}^{\prime} \ldots q_{m}^{\prime} R_{m}^{\prime}$, we define $\phi \sqsubseteq \phi^{\prime}$ iff there exists a monotonic injection $h: \bar{n} \rightarrow \bar{m}$ such that $q_{i}=q_{h(i)}^{\prime}$ for $i: 1 \leq i \leq n$ and the following conditions hold:

$$
\begin{aligned}
& -\left(R_{0}^{\prime} q_{1}^{\prime} \ldots q_{h(1)-1}^{\prime} R_{h(1)-1}^{\prime}\right)^{\bullet} \subseteq R_{0}^{\bullet} \\
& -\left(R_{h(i)}^{\prime} q_{h(i)+1}^{\prime} \ldots q_{h(i+1)-1}^{\prime} R_{h(i+1)-1}^{\prime}\right)^{\bullet} \subseteq R_{i}^{\bullet} \text { for } i: 1 \leq i \leq n-1 ; \\
& -\left(R_{h(n)}^{\prime} q_{h(n)+1}^{\prime} \ldots q_{m}^{\prime} R_{m}^{\prime}\right)^{\bullet} \subseteq R_{n}^{\bullet} .
\end{aligned}
$$

We have that $\phi_{1} \sqsubseteq \phi_{2}$ if and only if $\llbracket \phi_{2} \rrbracket \subseteq \llbracket \phi_{1} \rrbracket\left(\phi_{1}\right.$ is weaker than $\left.\phi_{2}\right)$.

Computing Predecessors. Given a set $S$ of CC's, it is possible to define a symbolic predecessor operator Pre that effectively computes, when applied to $S$, a set $S^{\prime}=\operatorname{Pre}(S)$ of CC's such that $\llbracket S^{\prime} \rrbracket$ is the set of configurations from which one can reach configurations in $\llbracket S \rrbracket$ using $\stackrel{*}{\longrightarrow}$ (i.e. predecessors).

We first introduce the symbolic predecessor computation for a $\forall_{L}$-rule, and then describe the case of the other transitions. Consider a transition $t$ of the form $q \rightarrow q^{\prime}: \forall_{L} P$ with $P \subseteq Q$. Then, $\operatorname{Pre}_{t}(\phi)$ is the set $\left\{\phi^{\prime} \mid \phi \leadsto_{t} \phi^{\prime}\right\}$ where $\sim_{t}$ is the minimal relation that satisfies one of the following conditions. Let $\phi=R_{0} q_{1} \ldots q_{n} R_{n}$ :

1. if there exists $i$ s.t. $q_{i}=q^{\prime}$ with $q_{j} \in P$ for each $j: 1 \leq j<i$, then $\phi \leadsto_{t}\left(R_{0} \cap P\right) q_{1} \ldots q_{i-1}\left(R_{i-1} \cap P\right) q R_{i} q_{i+1} \ldots q_{n} R_{n}$

2. if there exists $i$ s.t. $q^{\prime} \in R_{i}$ with $q_{j} \in P$ for each $j: 1 \leq j \leq i$, then $\phi \sim_{t}\left(R_{0} \cap P\right) q_{1} \ldots q_{i}\left(R_{i} \cap P\right) q R_{i} q_{i+1} \ldots q_{n} R_{n}$

Notice that: in (1) the length of the new basis and the number of contexts are the same as in $\phi$, whereas in the new constraint produced in (2) we add a new 
process as well as a new context. The case of $\forall_{R}$-rules is similar to that for $\forall_{L}$-rules. The remaining cases are given below.

Forall. Let $\phi=R_{0} q_{1} \ldots q_{n} R_{n}$. Consider a transition $t$ of the form $q \rightarrow q^{\prime}: \forall_{L R} P$ with $P \subseteq Q$. Then, $\sim_{t}$ is the minimal relation that satisfies one of the following conditions.

1. if there exists $i$ s.t. $q_{i}=q^{\prime}$ with $q_{j} \in P$ for each $j:(1 \leq j \neq i \leq n)$, then $\phi \sim_{t}\left(R_{0} \cap P\right) q_{1} \ldots q_{i-1}\left(R_{i-1} \cap P\right) q\left(R_{i} \cap P\right) q_{i+1} \ldots q_{n}\left(R_{n} \cap P\right)$.

2. if there exists $i$ s.t. $q^{\prime} \in R_{i}$ with $q_{j} \in P$ for each $j: 1 \leq j \leq n$, then $\phi \sim_{t}\left(R_{0} \cap P\right) q_{1} \ldots q_{i}\left(R_{i} \cap P\right) q\left(R_{i} \cap P\right) q_{i+1} \ldots q_{n}\left(R_{n} \cap P\right)$.

Local. Let $t$ be a local rule $q \rightarrow q^{\prime}, \sim_{t}$ is the minimal relation that satisfies one of the following conditions:

1. if there exists $E_{1}, E_{2} \in \mathbb{A}^{*}$ s.t. $\phi=E_{1} q^{\prime} E_{2}$, then $\phi \leadsto_{t} E_{1} q E_{2}$.

2. if there exists $E_{1}, E_{2} \in \mathbb{A}^{*}$ and $R \subseteq Q$ s.t. $\phi=E_{1} R E_{2}, q^{\prime} \in R$, and $q \notin R$, then $\phi \leadsto t E_{1} R q R E_{2}$.

Exist. Let $t$ be the rule $q \rightarrow q^{\prime}: \exists_{L} P, \sim_{t}$ is the minimal relation that satisfies one of the following conditions:

1. if there exists $E_{1}, E_{2}, E_{3} \in \mathbb{A}^{*}$ s.t. $\phi=E_{1} p E_{2} q^{\prime} E_{3}$, then $\phi \sim_{t} E_{1} p E_{2} q E_{3}$.

2. if there exists $E_{1}, E_{2}, E_{3} \in \mathbb{A}^{*}, R \subseteq Q$ s.t. $p \in R$, and $\phi=E_{1} R E_{2} q^{\prime} E_{3}$, then $\phi \sim_{t} E_{1} R p R E_{2} q E_{3}$.

3. if there exists $E_{1}, E_{2}, E_{3} \in \mathbb{A}^{*}, R \subseteq Q$ s.t. $p \in R, q^{\prime} \in R, q \notin R$ and $\phi=E_{1} p E_{2} R E_{3}$, then $\phi \sim_{t} E_{1} p E_{2} R q R E_{3}$.

4. if there exists $E_{1}, E_{2}, E_{3} \in \mathbb{A}^{*}, R, S \subseteq Q$ s.t. $p \in S, q^{\prime} \in R, q \notin R$ and $\phi=E_{1} S E_{2} R E_{3}$, then $\phi \sim_{t} E_{1} S p S E_{2} R q R E_{3}$.

5. if there exists $E_{1}, E_{2} \in \mathbb{A}^{*}, R \subseteq Q$ s.t. $p, q^{\prime} \in R, q \notin R$ and $\phi=E_{1} R E_{2}$, then $\phi \sim_{t} E_{1} R p R q R E_{3}$.

The rules for computing predecessors with respect to rules with $\exists_{R}, \exists_{L R}$ can be derived in a manner similar to the above described cases.

Symbolic Backward Reachability. Context expressions can be used for an exact representation of predecessor configurations. Each application of Pre is effectively computable. Let $\Phi_{0}$ be a set of CC's that represent an upward closed set of configurations (unsafe states). Starting from $\Phi_{0}$, we compute the sequence of sets of CC's-constraints $\Phi_{0}, \ldots, \Phi_{i}, \ldots$ such that $\Phi_{i+1}=\Phi_{i} \cup \bigcup_{t \in \mathcal{T}, \phi \in \Phi_{i}} \operatorname{Pre}_{t}(\phi)$. Each step of this sequence can be effectively computed. Furthermore, we can apply the entailment $\sqsubseteq$ to discharge CC's that do not add new information (i.e. stronger than an already computed constraint). If we reach a fixpoint at step $k$, then $\Phi_{k}$ gives us an exact representation of the predecessors of configurations in $\llbracket \Phi_{0} \rrbracket$. Thus, we can potentially use this fixpoint computation to solve PARCOV, i.e., to verify/falsify safety properties for configurations of arbitrary size. However, since our model is Turing complete (e.g. we can encode two counter machines using universally quantified conditions) the resulting CC's-based symbolic backward reachability analysis is not guaranteed to terminate. Therefore, in order to obtain a terminating verification procedure, we need to introduce some approximation. We discuss this point in the next section. 


\section{Approximated Context-Sensitive Symbolic Algorithm}

In this section, we present an approximated representation of context-sensitive constraints that we use to turn the (possibly non-terminating) CC-based verification procedure into an approximated verification algorithm. For this purpose, we first define a special class of constraints.

Definition 2. A simple context-sensitive (SCC-)constraint is a word in $\mathbb{A}^{*}$ of the form $R q_{1} R \ldots q_{n} R$ in which $\left\{q_{1}, \ldots, q_{n}\right\} \subseteq R \subseteq Q$.

Since the same constraint is uniformly applied to each context in the basis, we can simplify the notation and represent an SCC as a pair $(c, R)$, where $c \in Q^{*}$ and $c^{\bullet} \subseteq R \subseteq Q$. We refer to $R$ as the padding set. As we discuss later in this section, the requirement that the basis $c$ in included is the padding set has two consequences: it allows us to apply the theory of well-quasi ordering to ensure termination of the backward analysis (see Lemma 1); it gives us a natural way to define accelerations to speed up the symbolic computation of predecessors (see Section 4.1). Notice that an SCC need not represent an upward closed set of configurations. Indeed, the environment $R$ may be a strict subset of the set of all states. For instance, if $Q=\{a, b, c\}$ then the denotation of the SCC $(a a,\{a, b\})$ contains strings like $a a, a b a, a b a b, \ldots$ but it does not include any strings with $c$ even if they contain $a a$ as a substring (i.e. $a c a, a b a c, \ldots$ are not in its denotation).

A CC $\phi=R_{0} q_{0} \ldots q_{n} R_{n}$ can naturally be approximated by the following SCC:

$$
\phi^{\#}=\left(q_{0} \ldots q_{n}, \phi^{\bullet}\right)
$$

Indeed, it is immediate to check that $\llbracket \phi \rrbracket \subseteq \llbracket \phi^{\#} \rrbracket$. Let us now reconsider the symbolic operations (discussed in Section 3 for CC's) needed for implementing an SCC-based symbolic backward analysis.

Entailment. The entailment relation for SCC's can now be simplified as follows. For $\phi=(c, R)$ and $\phi^{\prime}=\left(c^{\prime}, R^{\prime}\right)$, we have that $\phi \sqsubseteq \phi^{\prime}$ iff $c \preceq c^{\prime}$ and $R \supseteq R^{\prime}$. We recall that $\phi \sqsubseteq \phi^{\prime}$ implies $\llbracket \phi^{\prime} \rrbracket \subseteq \llbracket \phi \rrbracket$.

Furthermore, we can prove that $\sqsubseteq$ is a Well Quasi-Ordering (WQO) for SCC's, i.e., for any infinite sequence $\phi_{0}, \phi_{1}, \phi_{2}, \ldots$, of constraints, there are $i<j$ such that $\phi_{i} \sqsubseteq \phi_{j}$. Indeed, let $\phi_{i}$ be of the form $\left(c_{i}, R_{i}\right)$. Since $Q$ is finite and $R_{i} \subseteq Q$ for all $i$, it follows that there is an infinite subsequence $\phi_{i_{0}}, \phi_{i_{1}}, \phi_{i_{2}}, \ldots$ such that $R_{i_{j}}=R_{i_{k}}$ for all $j, k$. By Higman's lemma [20] (which implies that $\preceq$ is a WQO on $\left.Q^{*}\right)$, there are $j<k$ such that $c_{i_{j}} \preceq c_{i_{k}}$, and hence $\phi_{i_{j}} \sqsubseteq \phi_{i_{k}}$. This gives the following lemma which we use later to prove termination of our algorithm.

Lemma 1. $\sqsubseteq$ is a WQO on the set of SCC's.

We extend the relation $\sqsubseteq$ to sets of SCC's such that $\Phi_{1} \sqsubseteq \Phi_{2}$ if for each $\phi_{2} \in \Phi_{2}$ there is a $\phi_{1} \in \Phi_{1}$ with $\phi_{1} \sqsubseteq \phi_{2}$. Notice that $\Phi_{1} \sqsubseteq \Phi_{2}$ implies that $\llbracket \Phi_{2} \rrbracket \subseteq \llbracket \Phi_{1} \rrbracket$.

As an example, consider the SCC $\phi=(p q,\{p, q, r\})$. Examples of configurations in $\llbracket \phi \rrbracket$ are $p r q$ and rprprqr. The set of bad states in Example 1 can be characterized by the SCC's $\left(q_{4} q_{4},\left\{q_{0}, q_{1}, q_{2}, q_{3}, q_{4}\right\}\right)$. Also, for the SCC $\phi=$ $(p q,\{p, q, r, s\})$ and $\phi^{\prime}=(q p p r q p,\{p, q, r\})$, we have $\phi \sqsubseteq \phi^{\prime}$. 


\subsection{Computing Predecessors}

The abstract predecessor operator Pre ${ }^{\#}$ is obtained as the composition of Pre and of the abstraction \#, i.e., $\operatorname{Pre} e^{\#}(\phi)=(\operatorname{Pre}(\phi))^{\#}$. However, it would be inefficient to implement it in this way. Indeed, in general Pre requires the analysis and generation of several cases (as for $\exists_{L}$-rules). As we discuss in Section 5, the large number of generated constraints makes the exact analysis unfeasible even on simple examples. For this reason, we show next how to directly define Pre as an operator working on SCC's-constraints.

First, we introduce some notations. For a basis $c$ and a state $q$, we write $c \otimes q$ to denote the set $\left\{c_{1} q c_{2} \mid c=c_{1} c_{2}\right\}$. The operation adds the singleton $q$ in an arbitrary position inside $c$. We define Pre $e^{\#}$ by means of a set of relations $\stackrel{t}{\sim}$ defined as follows. For a transition $t$, we define $\stackrel{t}{\sim}$ to be the smallest relation on constraints containing the following elements:

Local. If $t$ is a local transition of the form $q \rightarrow q^{\prime}$ then

$$
\begin{aligned}
& -\left(c_{1} q^{\prime} c_{2}, R\right) \stackrel{t}{\sim}\left(c_{1} q c_{2}, R \cup\{q\}\right) . \\
& -(c, R) \stackrel{t}{\rightarrow}\left(c_{1}, R \cup\{q\}\right) \text { if } q^{\prime} \in R \text { and } c_{1} \in(c \otimes q)
\end{aligned}
$$

In the first case, a process in the basis of the constraint performs a local transition from $q$ to $q^{\prime}$. We add $q$ to the padding set as required by the well-formedness of SCC's-constraints. From an operational perspective, augmenting the padding set with $q$ has an effect similar to widening operators used in relational analysis for unordered parameterized systems (e.g. based on polyhedra in [15]). To illustrate this, consider the rule $p \rightarrow q$ and the constraint $(r, R)$ where $R=\{q, r\}$. The exact predecessor computation would compute an infinite sequence of the form $R p R r R, R r R p R$ (one occurrence of $p$ ), $R r R p R p R, R p R r R p R, R p R p R r R$ (two occurrences of $p$ ), ... Our approximated operator computes the limit of the sequence, i.e., $(r p, R \cup\{p\}),(p r, R \cup\{p\})$ (at least one occurrence of $p)$. Thus, our abstraction plays here the role of a widening step for ordered configurations.

Exists. if $t$ is a global transition of the form $q \rightarrow q^{\prime}: \exists_{L} P$ then

$$
\begin{aligned}
- & \left(c_{1} q^{\prime} c_{2}, R\right) \stackrel{t}{\rightarrow}\left(c_{1} q c_{2}, R \cup\{q\}\right) \text { if } P \cap c_{1} \bullet \emptyset . \\
- & \left(c_{1} q^{\prime} c_{2}, R\right) \stackrel{t}{\rightarrow}\left(c_{3} q c_{2}, R \cup\{q\}\right) \text { if } p \in P \cap R, p \notin c_{1} \bullet, c_{3} \in\left(c_{1} \otimes p\right) . \\
- & \left(c_{1} p c_{2}, R\right) \stackrel{t}{\rightarrow}\left(c_{1} p c_{3}, R \cup\{q\}\right) \text { if } p \in P, q^{\prime} \in R, q \notin R, \text { and } c_{3} \in\left(c_{2} \otimes q\right) . \\
- & \left(c_{1} c_{2}, R\right) \stackrel{t}{\rightarrow}\left(c_{1} p c_{3}, R \cup\{q\}\right) \text { if } p \in P, p \notin c_{1} \bullet, q^{\prime} \in R, q \notin R, \text { and } c_{3} \in \\
& \left(c_{2} \otimes q\right) .
\end{aligned}
$$

In the first case, a process in the basis of the constraint performs an existential global transition from $q$ to $q^{\prime}$. The transition is performed if there is a witness which is to the left of the process and which is inside the basis of the constraint. The second case is similar to the first case, except that the witness is in the padding set (and not in the left part of the basis). Therefore, we add the witness explicitly in an arbitrary position to the left of the process. In the third case, a number of processes (at least one process) in the padding set perform the 
transition. There is a witness which enables the transition inside the basis. The witness should be to the left of the process making the transition. In the fourth case, both the witness and the process making the transition are in the padding set. This case is similar to the third case, except that we need to add the process making the transition explicitly in the basis. In a similar manner to the local transition case, we add $q$ to the padding to reflect the abstraction.

If $t$ is a global transition of the form $q \rightarrow q^{\prime}: \exists_{R} P$ or $q \rightarrow q^{\prime}: \exists_{L R} P$, then analogous conditions to the previous case hold.

Forall. $t$ is a global transition of the form $q \rightarrow q^{\prime}:{ }_{L R} P$, then

$$
\begin{aligned}
& -\left(c_{1} q^{\prime} c_{2}, R\right) \stackrel{t}{\rightarrow}\left(c_{1} q c_{2},(R \cap P) \cup\{q\}\right), \text { if }\left(c_{1} c_{2}\right)^{\bullet} \subseteq P . \\
& -\left(c_{1} c_{2}, R\right) \stackrel{t}{\rightarrow}\left(c_{1} q c_{2},(R \cap P) \cup\{q\}\right), \text { if } q^{\prime} \in R, q \notin R \text { and }\left(c_{1} c_{2}\right)^{\bullet} \subseteq P .
\end{aligned}
$$

In the first case, a process in the basis moves from $q$ to $q^{\prime}$. The remaining processes in the basis must be in $R$. Furthermore, we restrict the padding set to those processes within $R$. In the second case, a process of type $q$ in the padding set moves to $q^{\prime}$. Notice that in both cases, the state $q$ is added to the padding to reflect the abstraction.

If $t$ is a global transition of the form $q \rightarrow q^{\prime}: \forall_{L} P$, then

$$
\begin{aligned}
& -\left(c_{1} q^{\prime} c_{2}, R\right) \stackrel{t}{\sim}\left(c_{1} q c_{2}, R \cup\{q\}\right), \text { if } c_{1} \bullet \subseteq P . \\
& -\left(c_{1} c_{2}, R\right) \stackrel{t}{\sim}\left(c_{1} q c_{2}, R \cup\{q\}\right), \text { if } q^{\prime} \in R \text { and } c_{1} \subseteq P .
\end{aligned}
$$

In the first case, a process in the basis moves from $q$ to $q^{\prime}$. The remaining processes in the basis belong to $R$. In our constraints we use a single padding set to define the constraints on processes to the left and to the right of the process that makes the transition. Thus, to compute the precondition of the universal condition on the padding set we have to apply an over-approximation and use $R$ as constraints on contexts (processes to the left should be restricted to $R \cap P$ ). In the second case, a process $q$ from the padding set moves to $q^{\prime}$. Notice that in both cases, the state $q$ is added to the padding to reflect the abstraction. The second case is similar to the first case, except that the process that performs the transition is selected from the padding set.

If $t$ is a global transition of the form $q \rightarrow q^{\prime}: \forall_{R} P$, then analogous conditions to the previous case hold.

Now let $\leadsto:=\bigcup_{t \in T} \stackrel{t}{\rightarrow}$ and define for a constraint $\phi$ the set $(\phi \leadsto):=\left\{\phi^{\prime} \mid \phi \leadsto \phi^{\prime}\right\}$.

Lemma 2. For any constraint $\phi$, we have Pre $(\llbracket \phi \rrbracket) \subseteq \llbracket(\phi \leadsto) \rrbracket=\llbracket \operatorname{Pre}^{\#}(\phi) \rrbracket$.

Backward Reachability Algorithm. We use the relation $\leadsto$ to define a symbolic backward reachability algorithm for approximating solutions to PARCOV. We start with a finite set $\Phi_{F}$ of SCC's denoting $\widehat{C_{F}}$ (notice that we can always define SCC's that describe an upward-closed set). We generate a sequence $\Phi_{0} \sqsupseteq \Phi_{1} \sqsupseteq \Phi_{2} \sqsupseteq \cdots$ of finite sets of constraints such that $\Phi_{0}=\Phi_{F}$, and $\Phi_{j+1}=\Phi_{j} \cup\left(\Phi_{j} \leadsto\right)$. Since $\llbracket \Phi_{0} \rrbracket \subseteq \llbracket \Phi_{1} \rrbracket \subseteq \llbracket \Phi_{2} \rrbracket \subseteq \cdots$, the procedure terminates when we reach a point $j$ where $\Phi_{j} \sqsubseteq \Phi_{j+1}$. Thus, termination of the algorithm 
is guaranteed by Lemma 1. Notice that the termination condition implies that $\llbracket \Phi_{j} \rrbracket=\left(\bigcup_{0 \leq i \leq j} \llbracket \Phi_{i} \rrbracket\right)$. By Lemmas $2, \Phi_{j}$ denotes an over-approximation of the set of all predecessors of $\llbracket \Phi_{F} \rrbracket$. This means that if $\left(\right.$ Init $\left.\bigcap \llbracket \Phi_{j} \rrbracket\right)=\emptyset$, then there exists no $c \in \llbracket \Phi_{F} \rrbracket$ with Init $\stackrel{*}{\longrightarrow} c$. Thus, the algorithm can be used as a semi-test for checking PAR-COV.

Extensions. We discuss here possible extensions of the symbolic representation and of the model. The basic form of SCC's can be enriched in order to provide a more compact representation of sets of configurations. More specifically, as in [3, let us assume that individual processes have a state in $Q$ and a set of local Boolean variables in $V$. Let $\mathbb{B}$ be the set of Boolean formulas with predicates in $Q \cup V$. We can work on CC-constraints of the form $R_{0} b_{0} R_{1} \ldots b_{n} R_{n}$ $\left(\left(b_{0}, \ldots, b_{n}, R\right)\right.$ for SCC-constraints) where $b_{i}$ is a formula in $\mathbb{B}$ and $R_{i}(R)$ is a subset of formulas in $\mathbb{B}$. Now the basis describes a finite set of configurations with $n$ processes and each set $R_{i}$ gives constraints either on the state or on the local variables for processes occurring in the context. Furthermore, we can extend the exact/approximated symbolic computation of predecessors to rules with other synchronization mechanisms like broadcast communication and read/write operations on globally shared variables either with range in a finite domain or in the natural numbers. Operations on the latter type of shared variables can be obtained by using synchronization with special processes with state zero/one: increment is modelled via synchronization with a zero process that moves to one, decrement via synchronization with a one process that moves to zero, and zero test is modelled via a global condition "there are no processes with state one". The current value of the shared variable is the number of occurrence of processes in state one. Thus, this kind of variables may range over an unbounded set of natural numbers.

\section{Experimental Results}

We have implemented the verification procedures based on CC and SCC (see Table 11) and compared them to PFS (monotonic abstraction). To this purpose, we used examples of cache coherence protocols, mutual exclusion algorithms, and counter based synchronization problems. In the following, we briefly discuss some of the case studies.

The examples consist of the Illinois and the DEC Firefly cache coherence protocols from [15]; the Bakery and Burns mutual exclusion algorithms used in [3]; a compact model of Szymanski algorithm with atomicity conditions from 825, a refinement of Szymanski algorithm from [23] (see Fig. 21), and the GribomontZenner mutex from [18. Several synchronization and reference counting examples using unbounded integer counters are also considered. These include an abstract model of the reference counting example for page allocation in [16, and solutions to the readers/writers problem from [27] with priorities to readers or writers. We remark that in all examples global conditions are evaluated atomically. The results are summarized in Table 2. For each example, we give 
Table 1. Methods and tools listed in order of precision in the analysis

\begin{tabular}{|l|c|c|c|c|}
\hline Tool & Method & Approximation & Precision & Termination \\
\hline CC & backward reach. & none & exact & not guaranteed \\
\hline SCC & backward reach. & abstraction of CC's & over-approx & always guaranteed \\
\hline PFS & backward reach. & monotonic abst. & over-approx & always guaranteed \\
\hline
\end{tabular}

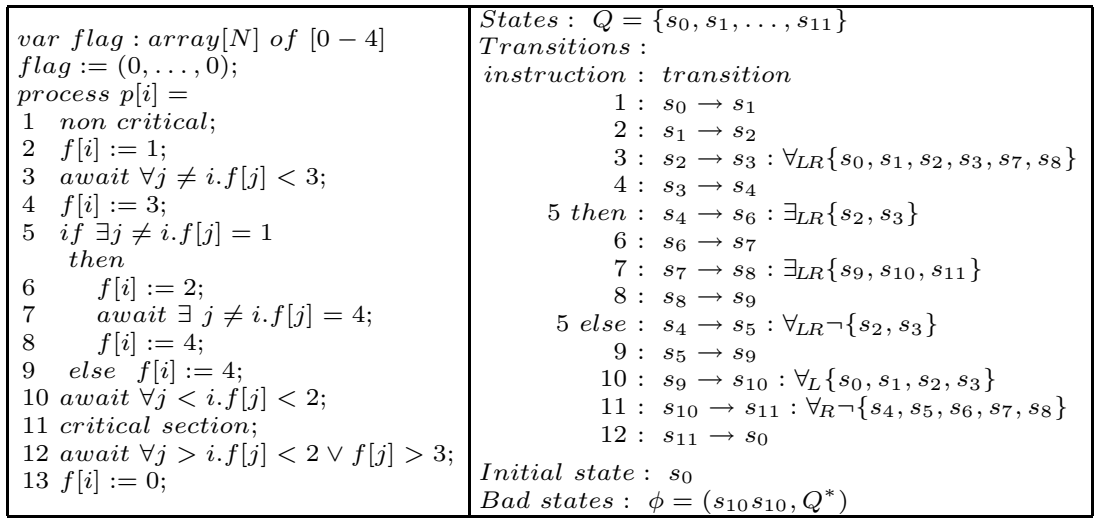

Fig. 2. Algorithm of Szymanski [23] (left), and its parameterized model (right)

the number of iterations performed by the reachability algorithm, the number of constraints upon termination of the algorithm, and the time (in seconds or minutes). We use _ in the appropriate fields to indicate that we had to stop the analysis after several hours.

In the simplest examples like the Bakery algorithm, each of the three methods (PFS,SCC,CC) automatically verifies mutual exclusion. However, exact analysis may diverge even on simple examples. Such a case occurs when testing mutual exclusion for the dirty cache line state in the DEC Firefly model of [15. A similar behavior was already observed with HyTech [19] (a tool manipulating polyhedra that can be used for unordered models) in [15]. In more complicated examples like the algorithms of Burns and Szymanski exact analysis does not terminate.

Monotonic abstraction proved to be precise for a wide range of parameterized systems $3,5,4,6,1$. However, it returned false positives for some of the protocols in Table 2. These are the fine grained formulations of Szymanski algorithm, the reference counting model, and particular versions of readers/writers. The main steps of the spurious error trace returned by PFS (monotonic abstraction) on the algorithm of Fig. 2 are described below.

$$
\begin{aligned}
& \left(s_{0}, s_{0}, s_{0}\right) \rightarrow^{*}\left(s_{1}, s_{1}, s_{1}\right) \rightarrow^{*}\left(s_{1}, s_{1}, s_{3}\right) \rightarrow\left(s_{2}, s_{1}, s_{3}\right) \rightarrow\left(s_{3}, s_{1}, s_{3}\right) \rightarrow\left(s_{3}, s_{1}, s_{4}\right) \\
& \rightarrow^{*}\left(s_{5}, s_{2}, s_{4}\right) \rightarrow\left(s_{9}, s_{2}, s_{4}\right) \rightarrow^{*}\left(s_{9}, s_{2}, s_{7}\right) \stackrel{\longrightarrow}{\longrightarrow}\left(s_{3}, s_{7}\right) \rightarrow^{*}\left(s_{10}, s_{10}\right)
\end{aligned}
$$


Table 2. Experimental results

\begin{tabular}{|c|c|c|c|c|c|c|}
\hline Model & Method & \# iter & \# constr & ex-time & spurious trace & verified \\
\hline \multirow[t]{3}{*}{ Bakery 3} & PFS & 2 & 2 & $0.01 \mathrm{~s}$ & & $\sqrt{ }$ \\
\hline & $\mathrm{CC}$ & 4 & 3 & $0.01 \mathrm{~s}$ & & $\sqrt{ }$ \\
\hline & SCC & 3 & 2 & $0.01 \mathrm{~s}$ & & $\sqrt{ }$ \\
\hline \multirow[t]{3}{*}{ Illinois 15} & PFS & 5 & 33 & $0.02 \mathrm{~s}$ & & $\sqrt{ }$ \\
\hline & $\mathrm{CC}$ & 2 & 17 & $0.05 \mathrm{~s}$ & & $\sqrt{ }$ \\
\hline & SCC & 7 & 53 & $0.18 \mathrm{~s}$ & & $\sqrt{ }$ \\
\hline \multirow[t]{3}{*}{ Burns [3] } & PFS & 14 & 40 & $0.05 \mathrm{~s}$ & & $\sqrt{ }$ \\
\hline & CC & - & -- & - & - & - \\
\hline & SCC & 15 & 48 & $0.02 \mathrm{~s}$ & & $\sqrt{ }$ \\
\hline \multirow[t]{3}{*}{ DEC Firefly 15} & PFS & 3 & 11 & $0.01 \mathrm{~s}$ & & $\sqrt{ }$ \\
\hline & CC & $\overline{--}$ & -- & -- & $\overline{--}$ & -- \\
\hline & SCC & 5 & 10 & $0.03 \mathrm{~s}$ & & $\sqrt{ }$ \\
\hline \multirow[t]{3}{*}{ Compact Szymanski $8 \mid 25$} & PFS & 10 & 17 & $0.1 \mathrm{~s}$ & & $\sqrt{ }$ \\
\hline & CC & - & - & - & - & - \\
\hline & SCC & 24 & 162 & $3.35 \mathrm{~s}$ & & $\sqrt{ }$ \\
\hline \multirow[t]{3}{*}{ Refined Szymanski 23} & PFS & 24 & 658 & $1.5 \mathrm{~s}$ & $\sqrt{ }$ & \\
\hline & CC & - & - & - & - & - \\
\hline & SCC & 34 & 641 & $1 \mathrm{~m}$ & & $\sqrt{ }$ \\
\hline \multirow[t]{3}{*}{ Gribomont-Zenner 18} & PFS & 36 & 197 & $0.2 \mathrm{~s}$ & $\sqrt{ }$ & \\
\hline & CC & - & - & -- & - & - \\
\hline & SCC & 56 & 863 & $5 \mathrm{~m}$ & & $\sqrt{ }$ \\
\hline \multirow[t]{3}{*}{ Ref. counting 16} & PFS & 7 & 15 & $0.02 \mathrm{~s}$ & $\sqrt{ }$ & \\
\hline & CC & -- & $\overline{--}$ & -- & -- & - \\
\hline & SCC & 7 & 8 & $0.01 \mathrm{~s}$ & & $\sqrt{ }$ \\
\hline \multirow{3}{*}{$\begin{array}{l}\text { Readers/writers }[27] \\
\text { (locks:no locks) }\end{array}$} & PFS & $(10: 5)$ & $(31: 28)$ & $(0.05 \mathrm{~s}: 0.02 \mathrm{~s})$ & $(: \sqrt{ })$ & $(\sqrt{ }:)$ \\
\hline & CC & -- & - & -- & -- & -- \\
\hline & SCC & $(7: 7)$ & $(12: 8)$ & $(0.02 \mathrm{~s}: 0.01 \mathrm{~s})$ & & $(\sqrt{ }: \sqrt{ })$ \\
\hline \multirow{3}{*}{$\begin{array}{l}\text { Readers/writers (locks:no locks) } \\
\text { refined, priority to readers }\end{array}$} & PFS & $(21: 7)$ & $(125: 67)$ & $(0.4 \mathrm{~s}: 0.6 \mathrm{~s})$ & $(: \sqrt{ })$ & $(\sqrt{ }:)$ \\
\hline & $\mathrm{CC}$ & -- & - & - & -- & - \\
\hline & SCC & $(25: 12)$ & $(128: 34)$ & $(1.7 \mathrm{~s}: 0.06 \mathrm{~s})$ & & $(\sqrt{ }: \sqrt{ })$ \\
\hline \multirow{3}{*}{$\begin{array}{l}\text { Readers/writers (locks:no locks) } \\
\text { refined, priority to writers }\end{array}$} & PFS & $(22: 9)$ & $(683: 219)$ & $(9.4 \mathrm{~s}: 0.3 \mathrm{~s})$ & $(: \sqrt{ })$ & $(\sqrt{ }:)$ \\
\hline & $\mathrm{CC}$ & -- & -- & -- & - & -- \\
\hline & SCC & $(27: 9)$ & $(646: 19)$ & (17.2s:0.03s) & & $(\sqrt{ }: \sqrt{ })$ \\
\hline \multirow[t]{3}{*}{ Light control [27] } & PFS & 13 & 96 & $0.06 \mathrm{~s}$ & $\sqrt{ }$ & \\
\hline & $C C$ & $\overline{--}$ & - & -- & - & - \\
\hline & SCC & 9 & 29 & $0.02 \mathrm{~s}$ & & $\sqrt{ }$ \\
\hline
\end{tabular}

The step indicated with $\longrightarrow_{0}$ corresponds to the deletion of a process violating the universal condition of the third instruction in Fig. 2(right). The spurious error trace is due to the fact that the denotation of the constraints manipulated by PFS contain every local state. When applied to this model, the approximated SCC-based algorithm terminates without detecting error traces, i.e., mutual exclusion is verified for the refined model for any number of processes. Notice that the compact model studied in 825, can be verified using both PFS and SCC.

\section{Conclusions and Related Work}

We have presented a new algorithm for parameterized verification based on special constraints, called SCC's, that retain approximated context-sensitive information on the type of processes executing in parallel with a finite set of completely specified individuals. We apply the new algorithm to several nontrivial examples in which other types of analysis fail. Furthermore, the new algorithm performs well on most of the examples that can be verified with existing 
parameterized verification techniques. In this paper, we consider protocols where variable updates are non-atomic. On the other hand, we consider in [4 models where global conditions are performed non-atomically. We plan to further investigate verification methods and efficient data structures for SCC's-like contextsensitive constraints that can help to lift the non-atomicity assumptions on both variable updates and global conditions.

Related Work. The constraints used for the exact analysis are similar to the APC regular expressions studied in [11. The verification method proposed in [11] is complementary to ours. Indeed, it is based on symbolic forward exploration with accelerations and without guarantying termination; whereas we consider here an over-approximation (based on simple context-sensitive constraints) that ensures the termination of symbolic backward exploration.

Other parameterized verification methods based on reductions to finite-state models have been applied to safety properties of mutual exclusion protocols like Szymanski's algorithm. Among these, we mention the invisible invariants method [824] and the environment abstraction method [1325]. In [25] environment abstraction is applied to a formulation of Szymanski with the same assumptions as the model in [8], called compact Szymanski in Table 2. This model can be verified using monotonic abstraction as discussed in Section 5. The refined model [23] we consider is different in that atomic instructions do not contain both tests and assignments. This potentially introduces new race conditions making verification a harder task. It is not clear whether the refined models of Szymanski's algorithm considered in the present paper can be automatically verified using the methods suggested in 813.

The infinite-state reference counting example we consider in this paper is inspired by a finite-state abstraction studied in [16]: in contrast to the predicateabstraction approach used in [16], we model reference counting for a physical page under observation via an unbounded integer shared variable, with increment, decrement, and zero-test.

Unordered models with counters can be modelled with systems working on unbounded integer variables such as in ALV [27/28] (based on the Omega library) and HyTech [19] (based on Halbwachs's polyhedra library). In these approaches extrapolation and widening operators are needed to enforce termination. This is typical for polyhedra-based methods when applied to models like DEC firefly and readers/writers. In contrast to methods like HyTech and ALV, the algorithm presented in this paper incorporates accelerations that can be applied both to ordered and unordered parameterized systems without losing termination.

\section{References}

1. Abdulla, P.A., Bouajjani, A., Cederberg, J., Haziz, F., Rezine, A.: Monotonic abstraction for programs with dynamic memory heaps. In: Gupta, A., Malik, S. (eds.) CAV 2008. LNCS, vol. 5123, pp. 341-354. Springer, Heidelberg (2008)

2. Abdulla, P.A., Čerāns, K., Jonsson, B., Tsay, Y.-K.: General decidability theorems for infinite-state systems. In: LICS 1996, pp. 313-321 (1996) 
3. Abdulla, P.A., Ben Henda, N., Delzanno, G., Rezine, A.: Regular model checking without transducers. In: Grumberg, O., Huth, M. (eds.) TACAS 2007. LNCS, vol. 4424, pp. 721-736. Springer, Heidelberg (2007)

4. Abdulla, P.A., Ben Henda, N., Delzanno, G., Rezine, A.: Handling parameterized systems with non-atomic global conditions. In: Logozzo, F., Peled, D.A., Zuck, L.D. (eds.) VMCAI 2008. LNCS, vol. 4905, pp. 22-36. Springer, Heidelberg (2008)

5. Abdulla, P.A., Delzanno, G., Rezine, A.: Parameterized verification of infinite-state processes with global conditions. In: Damm, W., Hermanns, H. (eds.) CAV 2007. LNCS, vol. 4590, pp. 145-157. Springer, Heidelberg (2007)

6. Abdulla, P.A., Delzanno, G., Haziza, F., Rezine, A.: Parameterized tree systems. In: Suzuki, K., Higashino, T., Yasumoto, K., El-Fakih, K. (eds.) FORTE 2008. LNCS, vol. 5048, pp. 69-83. Springer, Heidelberg (2008)

7. Abdulla, P.A., Jonsson, B., Nilsson, M., d'Orso, J.: Regular model checking made simple and efficient. In: Brim, L., Jančar, P., Křetínský, M., Kucera, A. (eds.) CONCUR 2002. LNCS, vol. 2421, pp. 116-130. Springer, Heidelberg (2002)

8. Arons, T., Pnueli, A., Ruah, S., Xu, J., Zuck, L.: Parameterized verification with automatically computed inductive assertions. In: Berry, G., Comon, H., Finkel, A. (eds.) CAV 2001. LNCS, vol. 2102, pp. 221-234. Springer, Heidelberg (2001)

9. Boigelot, B., Legay, A., Wolper, P.: Iterating transducers in the large. In: Hunt Jr., W.A., Somenzi, F. (eds.) CAV 2003. LNCS, vol. 2725, pp. 223-235. Springer, Heidelberg (2003)

10. Bouajjani, A., Habermehl, P., Vojnar, T.: Abstract regular model checking. In: Alur, R., Peled, D.A. (eds.) CAV 2004. LNCS, vol. 3114, pp. 372-386. Springer, Heidelberg (2004)

11. Bouajjani, A., Muscholl, A., Touili, T.: Permutation Rewriting and Algorithmic Verification. Inf. and Comp. 205(2), 199-224 (2007)

12. Bultan, T., Gerber, R., Pugh, W.: Model-checking concurrent systems with unbounded integer variables: symbolic representations, approximations, and experimental results. TOPLAS 21(4), 747-789 (1999)

13. Clarke, E., Talupur, M., Veith, H.: Environment abstraction for parameterized verification. In: Emerson, E.A., Namjoshi, K.S. (eds.) VMCAI 2006. LNCS, vol. 3855, pp. 126-141. Springer, Heidelberg (2005)

14. Dams, D., Lakhnech, Y., Steffen, M.: Iterating transducers. In: Berry, G., Comon, H., Finkel, A. (eds.) CAV 2001. LNCS, vol. 2102, pp. 286-297. Springer, Heidelberg (2001)

15. Delzanno, G.: Constraint-Based Verification of Parameterized Cache Coherence Protocols. FMSD 23(3), 257-301 (2003)

16. Emmi, M., Jhala, R., Kohler, E., Majumdar, R.: Verifying reference counted objects. In: Kowalewski, S., Philippou, A. (eds.) TACAS 2009. LNCS, vol. 5505, pp. 352-367. Springer, Heidelberg (2009)

17. Finkel, A., Schnoebelen, P.: Well-structured transition systems everywhere! TCS 256(1-2), 63-92 (2001)

18. Gribomont, E., Zenner, G.: Automated verification of Szymanski's algorithm. In: Steffen, B. (ed.) TACAS 1998. LNCS, vol. 1384, pp. 424-438. Springer, Heidelberg (1998)

19. Henzinger, T.A., Ho, P.-H., Wong-Toi, H.: HyTech: A Model Checker for Hybrid Systems. STTT 1, 110-122 (1997)

20. Higman, G.: Ordering by divisibility in abstract algebras. London Math. Soc. 2(7) (3), 326-336 (1952)

21. Kesten, Y., Maler, O., Marcus, M., Pnueli, A., Shahar, E.: Symbolic model checking with rich assertional languages. TCS 256, 93-112 (2001) 
22. Manna, Z., et al.: STEP: the Stanford Temporal Prover (1994)

23. Manna, Z., Pnueli, A.: An exercise in the verification of multi - process programs. Beauty is Our Business, 289-301 (1990)

24. Pnueli, A., Ruah, S., Zuck, L.: Automatic deductive verification with invisible invariants. In: Margaria, T., Yi, W. (eds.) TACAS 2001. LNCS, vol. 2031, pp. 82-97. Springer, Heidelberg (2001)

25. Talupur, M.: Abstraction techniques for parameterized verification. Ph.D thesis, CMU (2006)

26. Vardi, M.Y., Wolper, P.: An automata-theoretic approach to automatic program verification. In: LICS 1986, pp. 332-344 (1986)

27. Yavuz-Kahveci, T., Bultan, T.: A symbolic manipulator for automated verification of reactive systems with heterogeneous data types. STTT 5(1), 15-33 (2003)

28. Yavuz-Kahveci, T., Bultan, T.: Verification of parameterized hierarchical state machines using action language verifier. In: MEMOCODE 2005, pp. 79-88 (2005) 